\title{
Early alterations of cortical thickness and gyrification in migraine without aura: a retrospective MRI study in pediatric patients
}

Alessia Guarnera ${ }^{1,2}$, Francesca Bottino ${ }^{3}$, Antonio Napolitano ${ }^{3 *}$, Giorgia Sforza ${ }^{4}$, Marco Cappa ${ }^{5}$, Laura Chioma ${ }^{5}$, Luca Pasquini ${ }^{2,6}$, Maria Camilla Rossi-Espagnet ${ }^{1,2}$, Giulia Lucignani ${ }^{1}$, Lorenzo Figà-Talamanca ${ }^{1}$, Chiara Carducci ${ }^{1}$, Claudia Ruscitto ${ }^{7}$, Massimiliano Valeriani ${ }^{4,8}$, Daniela Longo ${ }^{1 \dagger}$ and Laura Papetti ${ }^{4 \dagger}$

\begin{abstract}
Background: Migraine is the most common neurological disease, with high social-economical burden. Although there is growing evidence of brain structural and functional abnormalities in patients with migraine, few studies have been conducted on children and no studies investigating cortical gyrification have been conducted on pediatric patients affected by migraine without aura.
\end{abstract}

Methods: Seventy-two pediatric patients affected by migraine without aura and eighty-two controls aged between 6 and 18 were retrospectively recruited with the following inclusion criteria: MRI exam showing no morphological or signal abnormalities, no systemic comorbidities, no abnormal neurological examination. Cortical thickness (CT) and local gyrification index (LGI) were obtained through a dedicated algorithm, consisting of a combination of voxel-based and surface-based morphometric techniques. The statistical analysis was performed separately on CT and LGI between: patients and controls; subgroups of controls and subgroups of patients.

Results: Patients showed a decreased LGI in the left superior parietal lobule and in the supramarginal gyrus, compared to controls. Female patients presented a decreased LGl in the right superior, middle and transverse temporal gyri, right postcentral gyrus and supramarginal gyrus compared to male patients. Compared to migraine patients younger than 12 years, the $\geq 12$-year-old subjects showed a decreased $C T$ in the superior and middle frontal gyri, pre- and post-central cortex, paracentral lobule, superior and transverse temporal gyri, supramarginal gyrus and posterior insula. Migraine patients experiencing nausea and/or vomiting during headache attacks presented an increased $C T$ in the pars opercularis of the left inferior frontal gyrus.

\footnotetext{
* Correspondence: antonio.napolitano@opbg.net

${ }^{\dagger}$ Daniela Longo and Laura Papetti contributed equally to this work.

${ }^{3}$ Medical Physics Department, Bambino Gesù Children's Hospital, Rome, Italy

Full list of author information is available at the end of the article
}

(C) The Author(s). 2021 Open Access This article is licensed under a Creative Commons Attribution 4.0 International License, which permits use, sharing, adaptation, distribution and reproduction in any medium or format, as long as you give appropriate credit to the original author(s) and the source, provide a link to the Creative Commons licence, and indicate if changes were made. The images or other third party material in this article are included in the article's Creative Commons licence, unless indicated otherwise in a credit line to the material. If material is not included in the article's Creative Commons licence and your intended use is not permitted by statutory regulation or exceeds the permitted use, you will need to obtain permission directly from the copyright holder. To view a copy of this licence, visit http://creativecommons.org/licenses/by/4.0/. The Creative Commons Public Domain Dedication waiver (http://creativecommons.org/publicdomain/zero/1.0/) applies to the data made available in this article, unless otherwise stated in a credit line to the data. 
Conclusions: Differences in CT and LGI in patients affected by migraine without aura may suggest the presence of congenital and acquired abnormalities in migraine and that migraine might represent a vast spectrum of different entities. In particular, $\geq 12$-year-old pediatric patients showed a decreased CT in areas related to the executive function and nociceptive networks compared to younger patients, while female patients compared to males showed a decreased $\mathrm{CT}$ of the auditory cortex compared to males. Therefore, early and tailored therapies are paramount to obtain migraine control, prevent cerebral reduction of cortical thickness and preserve executive function and nociception networks to ensure a high quality of life.

Keywords: migraine, aura, magnetic resonance imaging, cortical thickness, local gyrification index, cortical morphometry, cortical abnormalities, pediatric, phonophobia

\section{Background}

Migraine is the most common neurological disease [1$3]$ and ranks among the most disabling medical conditions, according to the WHO (World Health Organization) [4], with significant socio-economic burden[5].

Despite its high frequency, migraine pathophysiology and structural-functional features are far from being fully understood[6].

There is growing evidence that migraine may be a progressive disorder and cause brain structural and functional alterations [7-9]. Therefore, several Magnetic Resonance studies on these abnormalities have been conducted during the last two decades. Literature has mainly focused on adult populations $[6,9]$ and many studies have been conducted on small cohort samples, frequently with no distinction among the different migraine subtypes[10].

Migraine is a complex disorder, that may be related to an intrinsic predisposition reflected by anomalies in cortical gyrification and disease-related processes acting on cortical thickness. Studies on children are required to clarify the precise onset and nature of underlying cortical changes [6,11].

The investigation of pediatric migraine has frequently been encouraged to improve our understanding of the different features of the disease in children, such as duration and associated symptoms[6, 9].

To the best of our knowledge, no studies on cortical gyrification index have been conducted on children affected by migraine without aura and no studies have investigated a possible correlation between various migraine symptoms and gyrification abnormalities in pediatric populations.

The main goals of our study were to: (1) identify different patterns of cortical thickness and gyrification in pediatric patients affected by migraine without aura compared to healthy controls; (2) investigate possible correlations between these MRI parameters and clinical and demographic characteristics in patients compared to healthy controls; (3) evaluate any correlations between these MRI parameters and clinical and demographic characteristics among subgroups of patients, to identify potential brain imaging biomarkers.

\section{Methods \\ Participants}

The study was conducted in accordance with the ethical standards of the Institutional Research Committee and in accordance with the Helsinki Declaration and subsequent amendments. Informed consent was obtained from the Patients or their tutors prior to the MRI examination.

Patients were retrospectively recruited by reviewing Bambino Gesù Hospital Imaging archive from the 1st of January 2018 to the 31st of October 2020 using "headache" and "migraine" as keywords.

Two radiologists, respectively with thirty and six years of experience, performed a double-blinded analysis of patients' MRIs and any disagreement was resolved through a consensus. We included a total of 201 patients aged between 6 and 18 years with a high quality MRI including 3D T1 MPRAGE (Magnetization Prepared Rapid Gradient Echo Imaging) sequence and showing no morphological or signal abnormalities.

An experienced neurologist reviewed patients' medical records. Exclusion criteria were as follows: abnormal neurological examination; migraine attack during MRI; migraine prophylactic therapy; migraine abortive therapy within $24 \mathrm{~h}$ before the exam; catamenial migraines; sleep disorders; special abilities (athletic or artistic); major systemic disorders (psychological, oncological, vascular or other); maternal pathologies during pregnancy.

Eighty patients suffering from migraine without aura were interviewed and visited by two neurologists to confirm the diagnosis of migraine without aura according to the International Classification of Headache (ICHD3) [12] and to collect additional data.

In an anonymized database the following patients' data were reported: demographic characteristics, laterality of migraine; migraine monthly days; personal and familiar history of migraine; pain intensity; associated symptoms (photophobia, phonophobia, nausea and vomiting). 
The final cohort of patients consisted of 72 patients aged between 6 and 18 years (F:42) affected by episodic migraine without aura with a high-quality MRI exam acquired during the inter-ictal phase. The quality check of MRI examinations excluded the presence of signal and/ or morphological abnormalities which may affect cortical gyrification index and thickness due to confounding factors such as WM lesions, which have been suggested to contribute to GM changes [13-15].

Eighty-two healthy subjects aged between 6 and 18 years (F:40) formed the control group with optimal age and sex matching to minimize variability $[16,17]$. These subjects presented a high quality MRI exam without morphological and signal abnormalities and were interviewed and visited by a neurologist before inclusion in the control group in order to exclude migraine and other neurological diseases. No familiarity for migraine and/or headache was reported among healthy controls.

\section{MRI protocol}

Patients and controls brain MRIs were performed on the same 3T scanner (Magnetom Skyra, Siemens, Erlangen, Germany) with a 32-channel brain coil (L-W-H: $440 \mathrm{~mm}$ $\times 330 \mathrm{~mm} \times 370 \mathrm{~mm})$ and the following protocol: axial turbo spin-echo T2 (TR $6380 \mathrm{~ms}$, TE 109ms, ST 3mm); coronal turbo spin-echo T2 (TR $6380 \mathrm{~ms}$, TE 109ms, FA $150^{\circ}$, ST $3 \mathrm{~mm}$ ); axial FLAIR (TR $9000 \mathrm{~ms}$, TE $81 \mathrm{~ms}$, TI $2500 \mathrm{~ms}$, FA $150^{\circ}$, ST $3 \mathrm{~mm}$ ); axial DWI (TR $6400 \mathrm{~ms}$, TE 98ms, FA $75^{\circ}$, ST $4 \mathrm{~mm}$ ); sagittal 3D T1 MPRAGE (TR $1570 \mathrm{~ms}$, TE $2.67 \mathrm{~ms}$, TI 900ms, FA $9^{\circ}$, ST $0.8 \mathrm{~mm}$ ).

\section{Data processing}

Data was pre-processed with FreeSurfer 5.3 software [18], using a standard automatic pipeline (i.e. recon-all) that sequentially performed skull stripping, noise, bias, intensity correction and transformation to TalairachTournoux space to produce grey matter (GM) and white matter (WM) segmentation. Particularly, the FreeSurfer automatic pipeline determined and tessellated the GMWM boundary to generate the inner cortical surface (white surface), by combining information from tissue intensity and neighborhood constraints. The outer surface (pial surface) was generated through the expansion of the white surface with a point-to-point correspondence. Moreover, the FreeSurfer automatic pipeline computed Cortical thickness (CT) for each subject as the average distance measured from each surface to the other, according to Fischl and Dale [19]. The reconstructed white and grey surfaces obtained were visually checked to verify and correct any algorithmic misinterpretation of gyri and sulci. Local gyrification indices (LGI) were computed vertex-wise over the entire cortex by using the novel approach proposed by Lyu et al. (https://github.com/ilwoolyu/LocalGyrificationIndex)
[20], which is based on adaptive kernel for quantification of the local cortical folding. This algorithm quantifies cortical gyrification based on a spatially-adaptive kernel, which incorporates neighboring gyral crowns and sulcal fundi.

For statistical purposes and visualization, each subject was registered on Fsaverage brain surfaces, widely used in children's and teenagers' studies [21-23]. Fsaverage surfaces are composed by 163,842 total vertices which represent an index of spatial resolution for analyses performed across the brain surface grid based.

\section{Statistical analysis}

Sample sizes were estimated a priori as indicated by Pardoe et al. [24]. In particular, 50 subjects for each sample are required to detect a $0.25 \mathrm{~mm}$ cortical thickness difference and 10 subjects per group are required to detect a $1 \mathrm{~mm}$ cortical thickness difference [24]. Power calculation was set at 0.8 and type I error was set at $\mathrm{p}<0.05$. Univariate analysis of variance was carried out with SPSS software (PAWS Statistics 18.0) to test between-group differences in demographic variables. The statistical analysis was performed separately on CT and LGI between: healthy subjects and patients; subgroups of healthy controls and subgroups of patients (Table 1). We tested group differences in cortical parameters distributions by CT and LGI vertex-wise value mapping on a common spherical coordinate system (i.e. fsaverage), using spherical transformation. Differences among groups were assessed using the permutation test (1000 permutations per test) based on $t$ statistics, performed with the Permutation Analysis of Linear Models (PALM) FSL package. Subjects age was used as covariate of no interest. Age was mean centered (across all subjects) by subtracting the overall mean age from each individual age. We also computed Threshold-Free Cluster Enhancement statistical maps, where the initial raw statistical images were enhanced using both the intensity of the data point and information from neighboring voxels[25]. Differences between groups were detected by thresholding at family-wise error (FWE), corrected at $p<0.05$.

\section{Results}

The main demographic and clinical characteristics of patients with migraine and healthy control subjects are summarized in Table 2. Neither sex distribution $(P=0.2)$ nor mean age $(P=0.2)$ significantly differed between patients with migraine and healthy control subjects. Results were corrected in order to avoid false positives $[10,16,17]$.

\section{Cortical Gyrification}

Patients showed a decreased gyrification index in the left superior parietal lobule and in the left inferior parietal 
Table 1 Statistical analysis performed on Cortical Thickness and Local Gyrification Index

\section{PATIENTS VS CONTROLS}

\begin{tabular}{|c|c|c|c|}
\hline Cohort 1 & $\mathbf{N}^{\circ}$ & Cohort 2 & $\mathbf{N}^{\circ}$ \\
\hline Patients & 72 & Controls & 82 \\
\hline Patients $<12$ y & 42 & Controls $<12$ y & 46 \\
\hline Patients $\geq 12 y$ & 30 & Controls $\geq 12 y$ & 36 \\
\hline \multicolumn{4}{|l|}{ SUBGROUPS OF CONTROLS } \\
\hline Cohort 1 & $\mathbf{N}^{\circ}$ & Cohort 2 & $\mathbf{N}^{\circ}$ \\
\hline$<12 y$ & 46 & $\geq 12 y$ & 36 \\
\hline Females & 41 & Males & 41 \\
\hline \multicolumn{4}{|l|}{ SUBGROUPS OF PATIENTS } \\
\hline Cohort 1 & $\mathbf{N}^{\circ}$ & Cohort 2 & $\mathbf{N}^{\circ}$ \\
\hline Patients $<12 y$ & 42 & $\geq 12 y$ & 30 \\
\hline Females & 42 & Males & 30 \\
\hline Patients with $\mathrm{MMD}<5$ & 42 & Patients with $\mathrm{MMD} \geq 5$ & 30 \\
\hline Patients with photophobia and phonophobia & 41 & Patients with photophobia or phonophobia & 62 \\
\hline Patients with photophobia & 50 & Patients without photophobia & 22 \\
\hline Patients with nausea and/or vomiting & 38 & Patients without nausea and/or vomiting & 34 \\
\hline
\end{tabular}

The table illustrates the statistical analysis performed separately on cortical thickness and gyrification between: healthy patients and patients; subgroups of healthy controls and subgroups of patients. Cohort 1 and cohort 2 indicate the two groups of patients compared for each statistical analysis and $n^{\circ}$ indicates the number of patients forming a particular cohort.

MMD: migraine monthly days.

Table 2 Main demographic and clinical characteristics of healthy controls and patients affected by migraine without aura. Neither gender distribution $(P=0.2)$ nor mean age $(P=0.2)$ significantly differed between patients with migraine and healthy control subjects

\begin{tabular}{|c|c|c|}
\hline Characteristics & Healthy Controls & Patients \\
\hline $\mathrm{N}^{\circ}$ of Subjects & 82 & 72 \\
\hline$N^{\circ}$ of Subjects $<12$ years & 46 & 42 \\
\hline$N^{\circ}$ of Subjects $\geq 12$ years & 36 & 30 \\
\hline$N^{0} F / M$ & $41 / 41$ & $42 / 30$ \\
\hline Mean Age in years (STD) & $10.96(3.75)$ & $11.73(3.19)$ \\
\hline $\mathrm{N}^{\circ}$ of Subjects presenting $\mathrm{MMG}<5$ & - & 42 \\
\hline $\mathrm{N}^{\circ}$ of Subjects presenting $M M G \geq 5$ & - & 30 \\
\hline $\begin{array}{l}\text { Migraine Duration per attack } \\
\text { in hours ( } \mathrm{N}^{\circ} \text { of patients) }\end{array}$ & - & $<2$ h (26), <4 h (27), < 72 h (19) \\
\hline Months since first attack ( $n^{\circ}$ of patients) & - & $<6(3),<12(12),<24(13),<36(19),<72(25)$ \\
\hline Pain Intensity & - & mild (20), moderate (22), severe (30) \\
\hline$N^{\circ}$ of Subjects presenting Photophobia & - & 50 \\
\hline$N^{\circ}$ of Subjects presenting Phonophobia & - & 53 \\
\hline $\mathrm{N}^{\circ}$ of Subjects presenting Nausea & - & 35 \\
\hline $\mathrm{N}^{\circ}$ of Subjects presenting Vomiting & - & 16 \\
\hline $\mathrm{N}^{\circ}$ of Subjects presenting familiar cases of migraine & - & None (9), Mother (35), Father (7), Both parents (21) \\
\hline
\end{tabular}


lobule, particularly in the supramarginal gyrus, compared to healthy controls $(\mathrm{p}<0.05)$. We found a decreased gyrification index in the right superior, middle and transverse temporal gyri, in the right postcentral gyrus and in the right supramarginal gyrus in females compared to male patients $(\mathrm{p}<0.05)$ (Table 3). Statistical results including p-value maps are displayed on a common surface template in Fig. 1.

\section{Cortical Thickness}

Patients $\geq 12$-year-old showed decreased cortical thickness compared to younger ones, particularly involving: superior and middle frontal gyri, pre- and post-central cortex, paracentral lobule, superior and transverse temporal gyri, supramarginal gyrus and posterior insula. Migraine patients experiencing nausea and/or vomiting during headache attacks presented an increased cortical thickness in the pars opercularis of the inferior frontal gyrus (Table 3). Statistical results including p-value maps are displayed on a common surface template in Fig. 2.

\section{Discussion}

We found abnormalities in local gyrification index and cortical thickness in patients affected by migraine without aura compared to healthy controls and among patient subgroups. This evidence supports the idea of migraine being a complex pathology, possibly related to

Table 3 Results obtained from the statistical analysis performed on Cortical Thickness and Local Gyrification Index

\section{LOCAL GYRIFICATION INDEX}

\section{Patients vs. Patients $<$ Controls} Controls

$\begin{array}{ll} & \text { Left supramarginal gyrus } \\ & \text { Left superior parietal gyrus } \\ & \text { Left inferior parietal gyrus } \\ \text { Subgroups } & \text { Females < Males } \\ \text { of Patients } & \\ & \text { Right postcentral gyrus } \\ & \text { Right supramarginal gyrus } \\ & \text { Right superior temporal gyrus } \\ & \text { Right middle temporal gyrus } \\ & \text { Right transverse temporal gyrus }\end{array}$

\section{CORTICAL THICKNESS}

\section{Subgroups Patients $<12 y>$ Patients $\geq 12 y$ \\ of Patients}

$$
\begin{aligned}
& \text { Left superior frontal gyrus } \\
& \text { Left middle frontal gyrus } \\
& \text { Left precentral gyrus } \\
& \text { Left postcentral gyrus } \\
& \text { Left paracentral lobule } \\
& \text { Left superior temporal gyrus } \\
& \text { Left transverse temporal gyrus } \\
& \text { Left supramarginal gyrus } \\
& \text { Left posterior insula }
\end{aligned}
$$

Patients with nausea and/or vomiting > Patients without nausea and/or vomiting

Left pars opercularis $\mathrm{n}^{\circ}$ Vertices $p$

$1262 \quad 0.01$

$1014 \quad 0.01$

$3.53(2.40)$

$3.68(2.57)$

$4.03(2.74)$

256

$\mathrm{n}^{\circ}$

Vertices

Mean

F Mean LGI (STD)

value

$187 \quad 0.05 \quad 3.40(1.81)$

$3.25(1.79)$

$888 \quad 0.05$

$724 \quad 0.04$

$3.41(2.09)$

$3.95(2.67)$

$111 \quad 0.05$

0.04

$3.30(2.01)$

Vertices

Mean

$p$

Patients < 12y Mean CT (STD) in $\mathrm{mm}$

value

356

0.02

$2.43(0.98)$

$2.78(0.67)$

$2.74(0.73)$

$2.67(0.74)$

$2.61(0.85)$

$2.73(0.74)$

$2.66(0.78)$

$2.71(0.80)$

2.64 (0.74)

3610.03

Patients without nausea and/or

$\mathrm{n}^{\circ} \quad$ Mean

Vertices $p$

113 value

vomiting Mean CT (STD) in $\mathrm{mm}$

$0.04 \quad 2.52(0.94)$
Patients Mean LGI (STD)

$3.51(2.33)$

$3.65(2.52)$

$4.00(2.66)$

M Mean LGI (STD)

$3.49(1.88)$

$3.30(1.87)$

$3.47(2.17)$

$4.04(2.75)$

$3.37(2.09)$

Patients $\geq 12 y$ Mean CT

(STD)

$2.36(0.96)$

$2.71(0.65)$

$2.67(0.71)$

$2.61(0.72)$

$2.54(0.83)$

$2.65(0.72)$

$2.58(0.76)$

$2.64(0.79)$

$2.56(0.72)$

Patients with nausea and/ or vomiting Mean CT (STD)

$2.48(0.92)$

The table illustrates the results from statistical analysis performed separately on cortical thickness and gyrification between: healthy patients and patients; subgroups of healthy controls and subgroups of patients.

LGI: local gyrification index; CT: cortical thickness; mm: millimetres. 


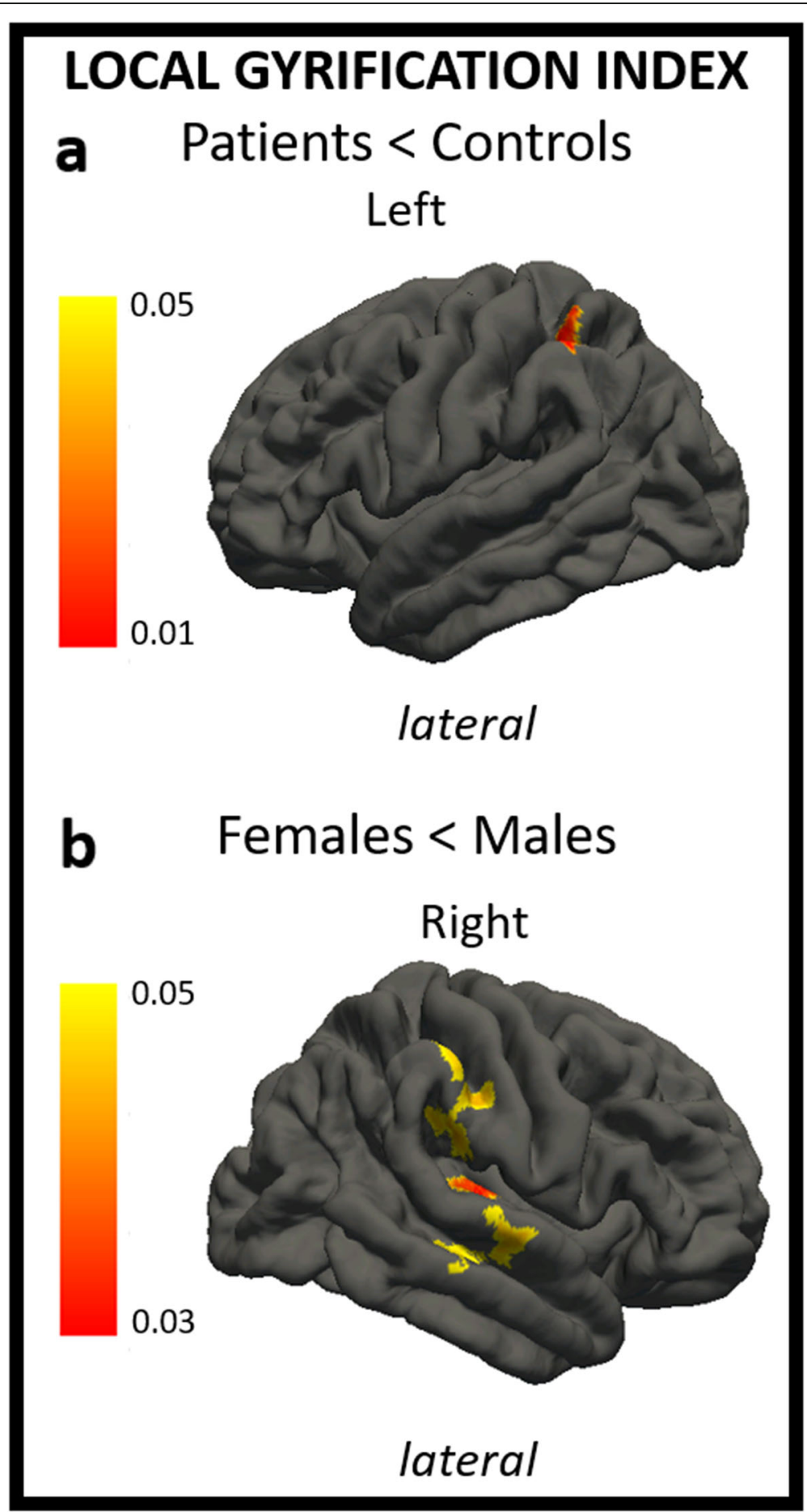

Fig. 1 The figure shows differences in local gyrification index in patients compared to healthy controls (A) and in female patients compared to male patients (B). In a, regions of decreased local gyrification index in patients vs. controls are shown through a color scale ranging from yellow $(p<0.05)$ to red $(p<0.01)$. In $\mathrm{b}$, regions of decreased local gyrification index in females vs. males are shown through a color scale ranging from yellow $(p<0.05)$ to red $(p<0.03)$. Only the most representative views are shown.

congenital and acquired brain abnormalities presenting from a very young age.

The working theory describes migraine pathogenesis as related to hyperexcitability of the trigeminovascular system. Specifically, cortical spreading depressions may promote the release of molecular mediators in the dura resulting in meningeal inflammation and sensory excitation of trigeminal afferences. This 


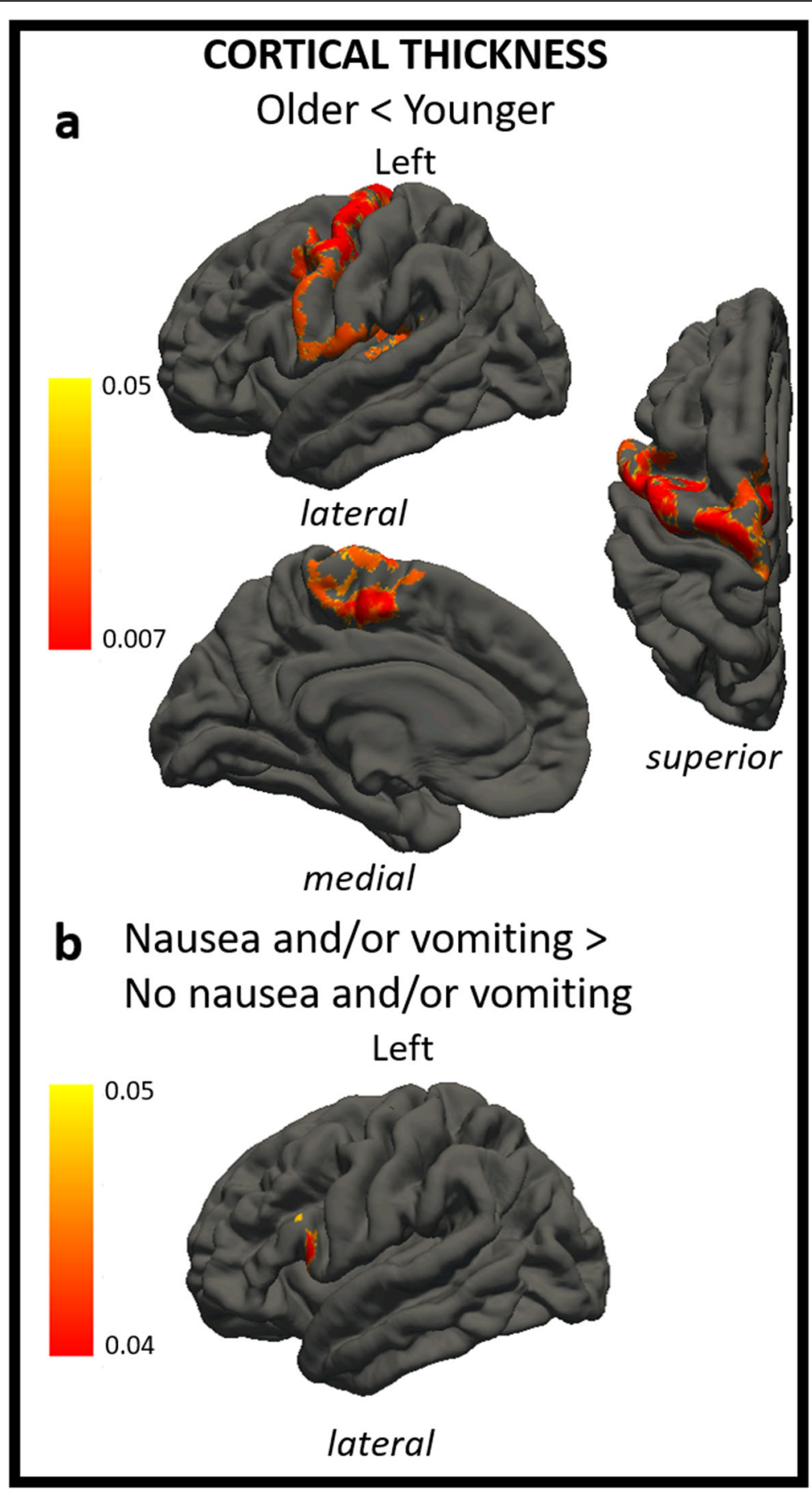

Fig. 2 The figure shows differences in cortical thickness in $\geq 12$-year-old patients compared to $<12$-year-old patients (A) and in patients with nausea and/or vomiting compared to patients without nausea and/or vomiting (B). In a, regions of decreased cortical thickness in $\geq 12$-year-old patients vs. $<12$-year-old patients are shown through a colour scale ranging from yellow $(p<0.05)$ to red $(p<0.007)$. In $b$, regions of increased cortical thickness in patients with nausea and/or vomiting vs. patients without nausea and/or vomiting are shown through a colour scale ranging from yellow $(p<0.05)$ to red $(p<0.007)$. Only the most representative views are shown.

nociceptive information is then projected on multiple cortical areas through central brain regions, leading to migraine symptoms [26].
Although its specific role in these processes is far from being fully understood, the cortex has been linked to the modulation and representation of pain in migraine as well as to the amplification of sensory inputs [27]. 
Neuroimaging studies have demonstrated that migraine patients present functional and morphological abnormalities compared to controls in relation to variables such as attack frequency or migraine attack duration, suggesting a possible link between anatomical and brain changes [28-30]. In particular, neuroimaging studies demonstrated macroscopic and microscopic changes in the brain of migraine patients, together with functional networks modifications [31].

\section{Local Gyrification Index}

Cortical gyrification is a complex process, which mainly takes place during the late fetal development and consists in cerebral cortex folding to allow cortical surface increase [11, 32, 33]. As it remains essentially stable during life, differences in cortical gyrification may reflect a congenital predisposition to develop migraine.

Despite the crucial information that cortical gyrification may offer, only two studies have investigated gyrification index changes in small cohorts of adults affected by migraine without aura $[5,10]$. In particular, Zhang et al. [5] identified an increased gyrification index in left postcentral gyrus, superior parietal lobule and right lateral occipital cortex, and a decreased gyrification index in the left rostral middle frontal gyrus compared with controls, while no differences have been identified by Masson et al. [10]. Moreover, Rieder et al. [34] investigated cortical alterations in medication-overuse headache in adults and observed higher LGI in two clusters extending respectively from the fusiform gyrus to adjacent medial temporal regions and in the occipital pole, this last being a good predictor for poor response after detoxification. On the other hand, Lai et al. [35] did not find significant differences between patients affected by chronic migraine and controls. These results, particularly from the study of Rieder et al. [34], may suggest a neurodevelopmental component to migraine disease chronicization or genetic predisposition to a more severe disease type.

\section{Patients vs. healthy controls}

We found a decreased gyrification index in the left superior parietal lobule and in the left inferior parietal lobule, particularly in the supramarginal gyrus, of patients compared to healthy controls.

The superior and the inferior parietal lobule are included in the [36] executive control network, which mainly involves fronto-striatal-parietal brain regions. Executive functions represent a complex subgroup of cognitive functions, which ensure a finalized behavior in multiple-choice contexts [36-38]. In particular, the superior parietal lobule has a pivotal role in task-switching, set-shifting and in the integration of information [39], while the inferior parietal lobule monitors selective attention [36]. The finding of a decreased gyrification index in the superior and inferior parietal lobule could explain the executive function deficits found in migraine patients during both ictal and interictal phases [37].

The superior and the inferior parietal lobule play a key role in the nociceptive pathway, particularly, the primary and secondary somatosensory cortices form the lateral pain system. Also, they add sensory-discrimination properties to pain processing by encoding location, intensity, and quality of pain [40-42]. This information is projected to the posterior parietal cortex which provides modulatory influences and conveys nociceptive information to widespread cortical brain regions [3, 42, 43]. The supramarginal gyrus is specifically involved in the cognitive evaluation of pain $[44,45]$ and a reduced pain-related activity of the supramarginal gyrus has been reported in headache patients with medication overuse [46].

The presented results confirm the longitudinal study of Liu.et al. [3], which assessed GM changes at baseline and after a follow-up of 1 year, showing decreased GM in the superior parietal gyrus, inferior parietal gyrus and supramarginal gyrus of migraine patients at follow-up. Structural changes in inferior parietal lobule and supramarginal gyrus match functional abnormalities identified in chronic migraine patients in a fMRI study by Chiapparini et al. [42] and functional alterations and abnormal connectivity identified between these areas and the hypothalamus in an fMRI study on cluster-headache by Ferraro et al. [46]. In particular, Chiapparini et al. tested brain areas activation during mechanical painful stimuli administration in chronic migraine patients and demonstrated significant activations in the inferior parietal cortex and in the supramarginal gyrus and reduced painrelated activity in the lateral pain pathway of patients with headache medication overuse [42].

These findings may suggest a role for these brain regions in migraine pain processing. In particular, an abnormal gyrification index in the superior and inferior parietal lobules may alter patients' pain sensorydiscrimination abilities and the complex modulatory mechanism taking part in pain processing, possibly disrupting the cognitive evaluation of pain.

\section{Female migraine vs. male migraine}

We found significantly decreased gyrification index in the right superior, middle and transverse temporal gyri, in the right postcentral gyrus and in the right supramarginal gyrus of females compared to male patients.

The search for a "sex specific phenotype" [47] of migraine is justified by the increased prevalence of migraine in females over 12 years [48, 49], differences in clinical symptoms and migraine presentation between the sexes $[50,51]$, the potential role of female hormones in migraine onset [52] and response to treatment [53]. 
Recent literature suggested that female and male migraine may be different in relation to specific functional and structural brain abnormalities compared to controls $[6,47,54-56]$.

As already discussed above, postcentral and supramarginal gyri are included in the nociceptive pathway. Previous studies investigating gray matter cortical thickness showed that females present an increased cortical thickness in areas associated with nociception, including the somatosensory cortex [54]. In particular, somatosensory cortical thickness has been demonstrated to be negatively correlated with response to migraine medications [55].

Although the role of the temporal lobes in pain processing is not completely understood and few studies are available in literature regarding temporal lobe abnormalities in migraine $[57,58]$, these areas are supposed to assign emotional valence to short-term memories related to painful experiences [59]. In particular, the superior temporal gyrus is involved in pain processing by supervising the mismatch between pain expectation and pain perception [60], pain anticipation [61], and pain expression [62]. In a fMRI study, Schwedt et al. [1] showed atypical connectivity between the middle temporal gyrus and widespread subcortical and cortical areas in migraine patients, possibly reflecting abnormal pain processing.

Our results confirm the hypothesis presented by Webb et al. [6], who suggested that structural abnormalities indicate the importance of sex in migraine.

Superior temporal gyrus and Heschl's gyrus, in particular Broadman areas $41-42$ and partially 22 , form the primary auditory cortex $[63,64]$. An increased volume of these gyri, as described by Aldemir et al. [57], may partially explain patient deficits in auditory stimuli processing, characterized by hypersensitivity and aversion to sound during migraine attacks, the onset of migraine attacks with auditory triggers, and the atypical sensory perception during the interictal phase $[65,66]$.

Differences in the gyrification index of the auditory cortex may justify different clinical presentations between male and female patients and why male patients with migraine more frequently present phonophobia compared to female ones $[48,50]$.

\section{Cortical Thickness}

CT undergoes dynamic changes through life in relation to normal development and diseases [11, 32, 33]. Since CT displays some variability due to physiologic and pathologic processes, changes induced by $\mathrm{mi}-$ graine appear realistic. Although cortical thickness in adult $[14,36,47]$ and pediatric $[11,58]$ migraine patients has been widely investigated, no "reliable brain morphological signature for migraine" [67] has been demonstrated so far.

\section{< 12-year-old Patients vs. $\geq 12$-year-old Patients with migraine}

We found cortical thickness to be significantly reduced in migraine patients $\geq 12$ years compared to those $<12$ years in: superior and middle frontal gyri, pre- and post-central cortex, paracentral lobule, superior and transverse temporal gyri, supramarginal gyrus and posterior insula.

Pediatric patients were divided in two subgroups in relation to age, since migraine prevalence of both sexes is almost equal up to age 12 and there are no sex-related differences, while after age 12 there is a significant female predominance, which progressively increases with age $[68,69]$.

A decreased cortical thickness in patients $\geq 12$ years may be justified by the older age and the longer duration of the disease compared to $<12$-years-old patients. This evidence is coherent with the inverse correlation [70, 71]between cortical thickness and age in migraine patients .

The finding of reduced cortical thickness of both frontal lobes in migraine patients is supported by several studies conducted on children [11, 13, 58] and adults $[14,36,72,73]$. As discussed above, this finding can be related to deficits in the executive control network during both ictal and interictal phases [37].

A decreased cortical thickness in the precentral, postcentral and supramarginal gyri confirms prior results obtained in studies conducted on adult migraine patients $[3,14,71,74]$. In particular, these cortical areas cooperate in pain processing by modulating the cognitive dimension of pain and by encoding the expected painful stimulus in relation to spatial location, intensity and quality of pain [14, 40-42, 75, 76]. The result obtained in the postcentral gyrus is supported by previous studies that show loss of volume and thinning of the cortex in patients with chronic pain of non-migraine origin [77, 78]. On the other hand, the superior temporal gyrus is involved in pain processing by supervising the mismatch between pain expectation and pain perception [60], and by regulating pain anticipation [61] and expression [62].

It has been suggested that frontal cortex reduction of cortical thickness is associated with the reorganization of the nociceptive network in relation to pain processing of migraine $[14,58,73]$. However, the temporal relation between migraine and cortical reduction of cortical thickness is not yet clear [6].

The identification of a thinner cortex in the superior and transverse temporal gyri may reflect the aforementioned deficits in auditory stimuli processing which are mostly appreciable during migraine attacks, but may or could persist in the interictal phase $[65,66]$.

The finding of a reduced cortical thickness in the posterior insula confirms prior literature. The pivotal role of insula in migraine and in the nociceptive network led to defining[80] insula as a "hub of activity" in migraine [79]. 
The insula represents a "multidimensional integration site for pain" since the nociceptive input received from the trigeminovascular pathway via the thalamus is first processed in the posterior insula, which encodes the intensity of pain [71, 81-84] and its anatomical location. From the posterior insula, the nociceptive input is conveyed to the anterior insula, which assigns emotional significance to the painful stimulus [79]. fMRI and PET studies have demonstrated posterior insula contribution to the sensory-discriminative aspects of pain processing [85-89], as it presents strong connectivity with the premotor, sensorimotor and supplementary motor areas and with the middle-posterior cingulate cortex $[79,90]$.

The posterior insula is also involved in interoceptive awareness, related to the internal state of migraine patients, which changes between the ictal and interictal phase [79, 91].

The finding of thinner insular cortex in patients $\geq 12$ years is supported by connectivity studies, as well as the evidence of structural-functional alterations in the pain processing network with aging. In particular, Dennis et al. [92] showed modifications in fiber density between the insula and various cortical regions from 12 to 30 years of age, while Ceko et al. [93] demonstrated a functional shift in insular connectivity in chronic pain states from being adaptive in < 12-year-old patients to being maladaptive in $\geq 12$-year-old ones. Insular changes with aging result in structural and functional alterations in pain processing $[79,94]$.

Accordingly, decreased cortical thickness of the posterior insula may reflect a structural-functional imbalance of brain homeostasis and may cause an altered perception and processing of pain [72, 95].

Insular involvement in migraine pathophysiology and the evidence of insular structural and functional alterations may pave the way to new and non-invasive treatment approaches in pediatric migraine. Particularly, aerobic exercise [96], stress limitation, maintenance of good hydration [97], optimal sleep hygiene [79], cognitive behavioral therapy along with pharmacotherapy have been proved extremely successful in migraine patients [79].

The importance of a tailored therapy roots in crucial differences between pediatric and adult migraine, as demonstrated by failure of standard adult treatment in the pediatric population $[6,98]$.

\section{Patients with nausea and/or vomiting vs. patients without nausea and/or vomiting}

Interestingly, patients experiencing nausea and/or vomiting during headache attacks showed increased cortical thickness in the left pars opercularis of the inferior frontal gyrus. The frontal operculum together with the insula represents the gustatory cortex, which is the cortical area dedicated to perceiving and distinguishing tastes [99]. Few studies identified abnormalities in cortical thickness of pars opercularis in patients affected by migraine and no paper has correlated changes in this area with migraine symptoms such as nausea or vomiting. In particular, Planchuelo-Gomez et al. [100] identified a significant negative correlation between attack duration in episodic migraine patients and gray matter volume in the right pars opercularis, while Hougaard et al. made a between-hemisphere comparison in migraine patients and identified an increased cortical thickness in the pars opercularis contralateral to the perceived headache side.

Harriott et al. [65] suggested that migraine patients experiment altered perception of painful and non-painful stimuli during ictal and interictal phases (unimodal special sensory processing in migraine patients) and present an abnormal integration of information from simultaneous and different sensory inputs (multisensory processing and integration).

Increased cortical thickness in the pars opercularis may reflect altered perception of stimuli and altered integration of information, leading to the experience of nausea and vomiting during migraine attacks.

Future longitudinal studies on larger cohorts are needed to confirm the presented results.

\section{Limitations}

The main limitation of our study is the retrospective design, which required us to select the study population from our Institution archive, including patients whose medical evaluation required a neuroimaging examination. To mitigate this limitation, we applied strict inclusion and exclusion criteria for patients and controls and interviewed and visited every subject before inclusion in the current study. Patients were not checked for anxiety or mood disorder symptoms with recognized standard scales. To limit this caveat, patients and families were interviewed to exclude physical and psychiatric comorbidities and sleep disorders.

\section{Conclusions}

The evidence of differences in cortical thickness and local gyrification index in patients compared to controls suggests that migraine is a complex pathology, possibly related to congenital and acquired brain abnormalities. In particular, $\geq 12$ year-old pediatric patients showed decreased cortical thickness in areas related to executive functions and nociceptive networks compared to $<12$-year-old patients, similar to what described in adult patients. Female patients compared to males showed decreased cortical thickness of the auditory cortex, which may justify the increased prevalence of phonophobia in males. Therefore, therapies should differ among 
patients depending on migraine features and individual characteristics.

\begin{abstract}
Abbreviations
MRI: magnetic resonance imaging; MPRAGE: Magnetization Prepared Rapid Gradient Echo Imaging; FLAIR: Fluid Attenuated Inversion Recovery; DWI: Diffusion Weighted Image; TR: repetition time; TE: echo time; TI: inversion time; FA: flip angle; ST: slice thickness; CT: cortical thickness; LGI: local gyrification index; Gl: gyrification index; WM: white matter; GM: grey matter; MMD: migraine monthly days; ICHD3: International Classification of Headache; WHO: World Health Organization; PALM: Permutation Analysis of Linear Models; FEW: Family Wise Error; STD: Standard Deviation
\end{abstract}

\section{Acknowledgements}

Not applicable.

\section{Authors' contributions}

$A G, D L, A N, M V, L P, F B$ have made substantial contributions to the conception and design of the work. AG, DL, MV, LP, GS, CR, MC, LC, MCRE, GL, LFT, CC have contributed to the acquisition and interpretation of data. AN, FB have contributed to the analysis and interpretation of data. AG has drafted the manuscript. DL, AN, MV, LP, FB, LP have substantially revised the manuscript. All the Authors have approved the submitted version. All the Authors have agreed both to be personally accounted for the author's own contribution and to ensure that questions related to the accuracy or integrity of any part of the work, even ones in which the author was not personally involved, are appropriately investigated, resolved, and the resolution documented in the literature.

\section{Funding}

The authors state that this work has not received any funding.

\section{Availability of data and materials}

Data and materials used and analyzed during the current study are available from the corresponding author upon reasonable request.

\section{Declarations}

\section{Ethics approval and consent to participate}

The study was conducted in accordance with the ethical standards of the Institutional Research Committee and in accordance with the Helsink Declaration and subsequent amendments. Institutional Review Board approval was waived because no new studies on human patients were undertaken specifically for this paper and no data from any individual person were presented. Informed consent was obtained from the Patients or their tutors prior to the MRI examination.

\section{Consent for publication}

Not applicable.

\section{Competing interests}

The authors of this manuscript declare no competing interest.

\footnotetext{
Author details

${ }^{1}$ Neuroradiology Unit, Imaging Department, Bambino Gesù Children's Hospital, IRCCS, Piazza S. Onofrio 4, 00165 Rome, Italy. ${ }^{2}$ Neuroradiology Unit, NESMOS Department, Sant'Andrea Hospital, La Sapienza University, Via di Grottarossa, 1035-1039, 00189 Rome, Italy. ${ }^{3}$ Medical Physics Department, Bambino Gesù Children's Hospital, Rome, Italy. ${ }^{4}$ Pediatric Headache Center, Bambino Gesù Children's Hospital, IRCCS, Piazza Sant'Onofrio 4, 00165 Rome, Italy. ${ }^{5}$ Unit of Endocrinology, Bambino Gesù Children's Hospital, IRCCS, Piazza Sant'Onofrio 4, 00165 Rome, Italy. ${ }^{6}$ Neuroradiology Service, Department of Radiology, Memorial Sloan Kettering Cancer Center, 10065 New York City, NY, USA. ${ }^{7}$ Child Neurology Unit, Systems Medicine Department, Tor Vergata University Hospital of Rome, 00133 Rome, Italy. ${ }^{8}$ Center for Sensory-Motor Interaction, Aalborg University, 9220 Aalborg, Denmark.
}

Received: 30 April 2021 Accepted: 5 July 2021

Published online: 22 July 2021

\section{References}

1. Schwedt TJ, Chong CD, Peplinski J, Ross K, Berisha V (2017 Aug) Persistent post-traumatic headache vs. migraine: an MRI study demonstrating differences in brain structure. J Headache Pain 22(1):87 18(

2. Schwedt TJ, Dodick DW (2009 Jun) Advanced neuroimaging of migraine. Lancet Neurol 8(6):560-568

3. Liu J, Lan L, Li G, Yan X, Nan J, Xiong S et al (2013 Dec) Migraine-related gray matter and white matter changes at a 1-year follow-up evaluation. J Pain 14(12):1703-1708

4. Leonardi M, Steiner TJ, Scher AT, Lipton RB (2005 Dec) The global burden of migraine: measuring disability in headache disorders with WHO's Classification of Functioning, Disability and Health (ICF). J Headache Pain 6(6):429-440

5. Zhang J, Wu Y-L, Su J, Yao Q, Wang M, Li G-F et al (2017 Dec) Assessment of gray and white matter structural alterations in migraineurs without aura. $J$ Headache Pain 18(1):74

6. Webb ME, Amoozegar F, Harris AD (2019 Nov) Magnetic Resonance Imaging in Pediatric Migraine. Can J Neurol Sci 46(6):653-665

7. Liu J, Zhao L, Li G et al (2012) Hierarchical alteration of brain structural and functional networks in female migraine sufferers. PLoS One 7:e51250

8. Jin C, Yuan K, Zhao L et al (2013) Structural and functional abnormalities in migraine patients without aura. NMR Biomed 26:58-64

9. Chong CD, Schwedt TJ, Dodick DW (2016) Migraine: What Imaging Reveals. Curr Neurol Neurosci Rep 16:64

10. Masson R, Demarquay G, Meunier D et al (2021) Is migraine associated to brain anatomical alterations? New data and coordinate-based meta-analysis. Brain Topogr 34:384-401

11. Messina R, Rocca MA, Colombo B et al (2013) Cortical abnormalities in patients with migraine: a surface-based analysis. Radiology 268:170-180

12. (2018) Headache Classification Committee of the International Headache Society (IHS) The International Classification of Headache Disorders, 3rd edition. Cephalalgia 38:1-211

13. Rocca MA, Ceccarelli A, Falini A et al (2006) Diffusion tensor magnetic resonance imaging at 3.0 tesla shows subtle cerebral grey matter abnormalities in patients with migraine. J Neurol Neurosurg Psychiatry 77: 686-689

14. Kim JH, Suh S-I, Seol HY et al (2008) Regional grey matter changes in patients with migraine: a voxel-based morphometry study. Cephalalgia 28 598-604

15. May A (2009) Morphing voxels: the hype around structural imaging of headache patients. Brain 132:1419-1425

16. Sheng L, Ma H, Shi Y et al (2020) Cortical Thickness in Migraine: A Coordinate-Based Meta-Analysis. Front Neurosci 14:600423

17. Datta R, Detre JA, Aguirre GK, Cucchiara B (2011) Absence of changes in cortical thickness in patients with migraine. Cephalalgia 31:1452-1458

18. Website. http://surfer.nmr.harvard.edu/. Accessed 18 Apr 2021

19. Fischl B, Dale AM (2000) Measuring the thickness of the human cerebral cortex from magnetic resonance images. Proc Natl Acad Sci U S A 97: 11050-11055

20. Lyu I, Kim SH, Bullins J et al (2017) Novel local shape-adaptive gyrification index with application to brain development. In: Medical Image Computing and Computer Assisted Intervention - MICCAI 2017. Springer International Publishing, Cham, pp 31-39

21. Peters S, Braams BR, Raijmakers MEJ et al (2014) The neural coding of feedback learning across child and adolescent development. J Cogn Neurosci 26:1705-1720

22. Liu P, Bai X, Pérez-Edgar KE (2019) Integrating high-density ERP and fMR measures of face-elicited brain activity in 9-12-year-old children: An ERP source localization study. Neurolmage 184:599-608

23. Schaer M, Ottet M-C, Scariati E et al (2013) Decreased frontal gyrification correlates with altered connectivity in children with autism. Front Hum Neurosci 7:750

24. Pardoe HR, Abbott DF, Jackson GD, Alzheimer's Disease Neuroimaging Initiative (2013) Sample size estimates for well-powered cross-sectional cortical thickness studies. Hum Brain Mapp 34:3000-3009

25. Mensen A, Khatami R (2013) Advanced EEG analysis using threshold-free cluster-enhancement and non-parametric statistics. Neuroimage 67:111-118 
26. Noseda R, Burstein R (2013) Migraine pathophysiology: anatomy of the trigeminovascular pathway and associated neurological symptoms, CSD, sensitization and modulation of pain. Pain 154 Suppl 1:. https://doi.org/10.1 016/.jpain.2013.07.021

27. Brennan KC, Pietrobon D (2018) A Systems Neuroscience Approach to Migraine. Neuron 97:1004-1021

28. Hubbard CS, Khan SA, Keaser ML et al (2014) Altered Brain Structure and Function Correlate with Disease Severity and Pain Catastrophizing in Migraine Patients. eNeuro 1:e20.14

29. Bashir A, Lipton RB, Ashina S, Ashina M (2013) Migraine and structural changes in the brain: A systematic review and meta-analysis. Neurology 81:1260-1268

30. Maniyar FH, Goadsby PJ (2013) Functional imaging in chronic migraine. Curr Pain Headache Rep 17:333

31. Coppola G, Parisi V, Di Renzo A, Pierelli F (2020) Cortical pain processing in migraine. J Neural Transm 127:551-566

32. Frye RE, Liederman J, Malmberg B et al (2010) Surface area accounts for the relation of gray matter volume to reading-related skills and history of dyslexia. Cereb Cortex 20:2625-2635

33. Kapellou O, Counsell SJ, Kennea N et al (2006) Abnormal cortical development after premature birth shown by altered allometric scaling of brain growth. PLoS Med 3:e265

34. Riederer F, Schaer M, Gantenbein AR et al (2017) Cortical Alterations in Medication-Overuse Headache. Headache 57:255-265

35. Lai K-L, Niddam DM, Fuh J-L et al (2020) Cortical morphological changes in chronic migraine in a Taiwanese cohort: Surface- and voxel-based analyses. Cephalalgia 40:575-585

36. Schmitz N, Arkink EB, Mulder M et al (2008) Frontal lobe structure and executive function in migraine patients. Neurosci Lett 440:92-96

37. Shallice T, Burgess PW (1991) Deficits in strategy application following frontal lobe damage in man. Brain 114(Pt 2):727-741

38. Robbins TW (2007) Shifting and stopping: fronto-striatal substrates, neurochemical modulation and clinical implications. Philos Trans R Soc Lond B Biol Sci 362:917-932

39. Fassbender C, Murphy K, Foxe JJ et al (2004) A topography of executive functions and their interactions revealed by functional magnetic resonance imaging. Brain Res Cogn Brain Res 20:132-143

40. Oshiro Y, Quevedo AS, McHaffie JG et al (2007) Brain mechanisms supporting spatial discrimination of pain. J Neurosci 27:3388-3394

41. Price DD (2002) Central Neural Mechanisms that Interrelate Sensory and Affective Dimensions of Pain. Mol Interventions 2:392-403

42. Chiapparini L, Ferraro S, Grazzi L, Bussone G (2010) Neuroimaging in chronic migraine. Neurol Sci 31(Suppl 1):S19-S22

43. Apkarian VA, Hashmi JA, Baliki MN (2011) Pain and the brain: specificity and plasticity of the brain in clinical chronic pain. Pain 152:S49-S64

44. Lamm C, Decety J, Singer T (2011) Meta-analytic evidence for common and distinct neural networks associated with directly experienced pain and empathy for pain. Neuroimage 54:2492-2502

45. Moulton EA, Pendse G, Becerra LR, Borsook D (2012) BOLD responses in somatosensory cortices better reflect heat sensation than pain. J Neurosci 32:6024-6031

46. Ferraro S, Grazzi L, Mandelli ML et al (2012) Pain processing in medication overuse headache: a functional magnetic resonance imaging (fMRI) study. Pain Med 13:255-262

47. Maleki N, Linnman C, Brawn J et al (2012) Her versus his migraine: multiple sex differences in brain function and structure. Brain 135:2546-2559

48. Özge A, Abu-Arafeh I, Gelfand AA et al (2017) Experts' opinion about the pediatric secondary headaches diagnostic criteria of the ICHD-3 beta. J Headache Pain 18:113

49. Stewart WF (1992) Prevalence of migraine headache in the United States. Relation to age, income, race, and other sociodemographic factors. JAMA: The Journal of the American Medical Association 267:64-69

50. Casucci G, Villani V, d'Onofrio F, Russo A (2015) Migraine and lifestyle in childhood. Neurol Sci 36(Suppl 1):97-100

51. Bigal ME, Ashina S, Burstein R et al (2008) Prevalence and characteristics of allodynia in headache sufferers: a population study. Neurology 70:1525-1533

52. Martin VT, Lee J, Behbehani MM (2007) Sensitization of the trigeminal sensory system during different stages of the rat estrous cycle: implications for menstrual migraine. Headache 47:552-563

53. Ferrari A, Tiraferri I, Neri L, Sternieri E (2011) Why pharmacokinetic differences among oral triptans have little clinical importance: a comment. J Headache Pain 12:5-12
54. Faria V, Erpelding $N$, Lebel $A$ et al (2015) The migraine brain in transition: girls vs boys. Pain 156:2212-2221

55. Hubbard CS, Becerra L, Smith JH et al (2016) Brain Changes in Responders vs. Non-Responders in Chronic Migraine: Markers of Disease Reversal. Front Hum Neurosci 10:497

56. Goffaux P, Girard-Tremblay L, Marchand S et al (2014) Individual differences in pain sensitivity vary as a function of precuneus reactivity. Brain Topogr 27:366-374

57. Aldemir A, Yucel K, Güven $\mathrm{H}$ et al (2020) Structural neuroimaging findings in migraine patients with restless legs syndrome. Neuroradiology 62:1301-1313

58. Rocca MA, Messina R, Colombo B et al (2014) Structural brain MRI abnormalities in pediatric patients with migraine. J Neurol 261:350-357

59. Godinho F, Magnin M, Frot M et al (2006) Emotional modulation of pain: is it the sensation or what we recall? J Neurosci 26:11454-11461

60. De Pauw R, Coppieters I, Caeyenberghs K et al (2019) Associations between brain morphology and motor performance in chronic neck pain: A wholebrain surface-based morphometry approach. Hum Brain Mapp 40:4266-4278

61. Palermo S, Benedetti F, Costa T, Amanzio M (2015) Pain anticipation: an activation likelihood estimation meta-analysis of brain imaging studies. Hum Brain Mapp 36:1648-1661

62. Budell L, Kunz M, Jackson PL, Rainville P (2015) Mirroring pain in the brain: emotional expression versus motor imitation. PLoS One 10:e0107526

63. Pickles JO (2012) An Introduction to the Physiology of Hearing. BRILL

64. Nakai Y, Jeong J-W, Brown EC et al (2017) Three- and four-dimensional mapping of speech and language in patients with epilepsy. Brain 140:1351-1370

65. Harriott AM, Schwedt TJ (2014) Migraine is associated with altered processing of sensory stimuli. Curr Pain Headache Rep 18:458

66. Vingen JV, Pareja JA, Støren O et al (1998) Phonophobia in migraine. Cephalalgia 18:243-249

67. Wang HZ, Wang WH, Shi HC, Yuan CH (2020) Is there a reliable brain morphological signature for migraine? J Headache Pain. 21(1):89. https://doi.org/10.1186/s10194-020-01158-7. PMID: 32652927; PMCID: PMC7353790

68. Stewart WF, Linet MS, Celentano DD et al (1991) Age- and sex-specific incidence rates of migraine with and without visual aura. Am J Epidemiol 134:1111-1120

69. Özge A, Faedda N, Abu-Arafeh I et al (2017) Experts' opinion about the primary headache diagnostic criteria of the ICHD-3rd edition beta in children and adolescents. J Headache Pain 18:109

70. Chong CD, Dodick DW, Schlaggar BL, Schwedt TJ (2014) Atypical agerelated cortical thinning in episodic migraine. Cephalalgia 34:1115-1124

71. Celle S, Créac'h C, Boutet C et al (2018) Elderly Patients with Ongoing Migraine Show Reduced Gray Matter Volume in Second Somatosensory Cortex. J Oral Facial Pain Headache 32:67-74

72. Dai Z, Zhong J, Xiao P et al (2015) Gray matter correlates of migraine and gender effect: A meta-analysis of voxel-based morphometry studies. Neuroscience 299:88-96

73. Hougaard A, Amin FM, Magon S et al (2015) No abnormalities of intrinsic brain connectivity in the interictal phase of migraine with aura. Eur I Neurol 22:702-746

74. Jia Z, Yu S (2017) Grey matter alterations in migraine: A systematic review and meta-analysis. Neuroimage Clin 14:130-140

75. Peyron R, García-Larrea L, Grégoire MC et al (1999) Haemodynamic brain responses to acute pain in humans: sensory and attentional networks. Brain 122(Pt 9):1765-1780

76. Peyron R, Laurent B, García-Larrea L (2000) Functional imaging of brain responses to pain. A review and meta-analysis (2000). Neurophysiol Clin 30: 263-288

77. Baliki MN, Schnitzer TJ, Bauer WR, Apkarian AV (2011) Brain morphological signatures for chronic pain. PLoS One 6:e26010

78. Frøkjær JB, Bouwense SAW, Olesen SS et al (2012) Reduced cortical thickness of brain areas involved in pain processing in patients with chronic pancreatitis. Clin Gastroenterol Hepatol 10:434-48.e1

79. Borsook D, Veggeberg R, Erpelding $\mathrm{N}$ et al (2016) The Insula: A "Hub of Activity" in Migraine. Neuroscientist 22:632-652

80. Brooks JCW, Tracey I (2007) The insula: a multidimensional integration site for pain. Pain 128:1-2

81. Peyron R (2016) Functional brain imaging: what has it brought to our understanding of neuropathic pain? A special focus on allodynic pain mechanisms. Pain 157(Suppl 1):S67-S71 
82. Segerdahl AR, Mezue M, Okell TW et al (2015) The dorsal posterior insula is not an island in pain but subserves a fundamental role - Response to: "Evidence against pain specificity in the dorsal posterior insula" by Davis et al. F1000Res 4:1207

83. Frot M, Magnin M, Mauguière F, Garcia-Larrea L (2007) Human SIl and posterior insula differently encode thermal laser stimuli. Cereb Cortex 17: 610-620

84. Apkarian AV, Baliki MN, Geha PY (2009) Towards a theory of chronic pain. Prog Neurobiol 87:81-97

85. Eck J, Richter M, Straube T et al (2011) Affective brain regions are activated during the processing of pain-related words in migraine patients. Pain 152 1104-1113

86. Bahra A, Matharu MS, Buchel C et al (2001) Brainstem activation specific to migraine headache. Lancet 357:1016-1017

87. Demarquay G, Lothe A, Royet JP et al (2011) Brainstem changes in 5-HT1A receptor availability during migraine attack. Cephalalgia 31:84-94

88. Kim JH, Kim S, Suh S-I et al (2010) Interictal metabolic changes in episodic migraine: a voxel-based FDG-PET study. Cephalalgia 30:53-61

89. Shin JH, Kim YK, Kim H-J, Kim J-S (2014) Altered brain metabolism in vestibular migraine: comparison of interictal and ictal findings. Cephalalgia 34:58-67

90. Cauda F, D'Agata F, Sacco K et al (2011) Functional connectivity of the insula in the resting brain. Neuroimage 55:8-23

91. Craig AD (2013) An interoceptive neuroanatomical perspective on feelings, energy, and effort. Behav Brain Sci 36:685-686; discussion 707-26

92. Dennis EL, Jahanshad N, McMahon KL et al (2014) Development of insula connectivity between ages 12 and 30 revealed by high angular resolution diffusion imaging. Hum Brain Mapp 35:1790-1800

93. Ceko M, Bushnell MC, Fitzcharles M-A, Schweinhardt P (2013) Fibromyalgia interacts with age to change the brain. Neuroimage Clin 3:249-260

94. Tseng M-T, Chiang M-C, Yazhuo K et al (2013) Effect of aging on the cerebral processing of thermal pain in the human brain. Pain 154:2120-2129

95. Borsook D, May A, Goadsby PJ, Hargreaves R (2012) The Migraine Brain: Imaging Structure and Function. Oxford University Press

96. Lockett D-M The effects of aerobic exercise on migraine

97. Ostfeld AM, Wolff HG (1955) Arterenol (norepinephrine) and vascular headache of the migraine type; studies on headache. AMA Arch Neurol Psychiatry 74:131-136

98. Powers SW, Coffey CS, Chamberlin LA et al (2017) Trial of Amitriptyline, Topiramate, and Placebo for Pediatric Migraine. N Engl J Med 376:115-124

99. Obiefuna S, Donohoe C (2020) Neuroanatomy, Nucleus Gustatory. In: StatPearls. StatPearls Publishing, Treasure Island (FL)

100. Planchuelo-Gómez Á, García-Azorín D, Guerrero ÁL et al (2020) Gray Matter Structural Alterations in Chronic and Episodic Migraine: A Morphometric Magnetic Resonance Imaging Study. Pain Med 21:2997-3011

\section{Publisher's Note}

Springer Nature remains neutral with regard to jurisdictional claims in published maps and institutional affiliations.

Ready to submit your research? Choose BMC and benefit from:

- fast, convenient online submission

- thorough peer review by experienced researchers in your field

- rapid publication on acceptance

- support for research data, including large and complex data types

- gold Open Access which fosters wider collaboration and increased citations

- maximum visibility for your research: over $100 \mathrm{M}$ website views per year

At $\mathrm{BMC}$, research is always in progress.

Learn more biomedcentral.com/submissions 